\title{
A specialist nurse intervention reduced readmissions in patients admitted to hospital with chronic heart failure
}

Blue L, Lang E, McMurray JJ, et al. Randomised controlled trial of specialist nurse intervention in heart failure. BMJ 2001 Sep 29;323:715-8.

\section{QUESTION: Can a specialist nurse intervention reduce mortality and morbidity in patients admitted to hospital with chronic heart failure?}

\section{Design}

Randomised (allocation concealed*), blinded \{outcome assessors $\} \nmid, *$ controlled trial with 1 year of follow up.

\section{Setting}

An acute medical admissions unit at a teaching hospital in Glasgow, Scotland, UK.

\section{Patients}

165 patients (mean age 75 y, 58\% men) who were admitted on an emergency basis with heart failure caused by left ventricular systolic dysfunction. Exclusion criteria were inability to comply with the intervention, acute myocardial infarction, comorbidity likely to lead to death or readmission in the near future, planned discharge to long term residential care, or residence outside of the hospital catchment area. Follow up at 1 year was $95 \%$.

\section{Intervention}

84 patients were allocated to usual care plus a specialist nurse intervention, which consisted of planned home visits of decreasing frequency supplemented by telephone contact as needed. The aim was to educate patients about heart failure and its treatment, optimise treatment, monitor electrolyte concentrations, teach self monitoring and management, encourage treatment adherence, liaise with other healthcare providers, and provide psychological support. Nurses were given training and followed written protocols on the use of specific drugs. Patients were given a pocket-sized booklet that included information about heart failure and its treatment; contact information for nurses; a list of their drugs, weight, and blood test results; and details of planned visits. 81 patients were allocated to usual care and managed by the admitting physician and subsequently the general practitioner. They did not see the specialist nurses after discharge.

\section{Main outcome measures}

Combined outcome of death or readmission for heart failure (emergency or elective). Secondary outcomes included combined death or readmission for any reason, death, readmission for worsening chronic heart failure, and readmission for any reason.

\section{Main results}

At 1 year, fewer patients in the specialist nurse group than in the usual care group had the combined outcome of death or readmission for heart failure, and fewer were readmitted for heart failure (table). The groups did not differ for combined death or readmission for any reason $(62 \%$ v $75 \%$, $\mathrm{p}=0.075)$, death $(30 \%$ v $31 \%, \mathrm{p}=0.81)$, or for readmission for any reason $(56 \% v 60 \%, \mathrm{p}=0.27)$.

\section{Conclusion}

A specialist nurse intervention reduced readmissions for heart failure in patients admitted to hospital with chronic heart failure.

*See glossary.

†Information provided by author.
Source of funding. Scottish Office, Department of Health.

For correspondence: $D$ JJ McMurray, Western Infirmary, Glasgow, Scotland, UK.

j.mcmurray@ bio.gla.ac.uk.

A modified version of this abstract also appears in Evidence-Based Nursing.

A specialist nurse intervention v usual care for chronic heart failure $(H F)+$

\begin{tabular}{lllll} 
Outcomes at 1 year & Intervention & Usual care & RRR (95\% CI) & NNT (CI) \\
Death or readmission for HF & $37 \%$ & $53 \%$ & $30 \%(3$ to 53$)$ & $7(4$ to 70$)$ \\
\hline Readmission for HF & $14 \%$ & $32 \%$ & $57 \%(21$ to 78$)$ & $6(5$ to 16$)$
\end{tabular}

†Abbreviations defined in glossary; RRR, NNT, and Cl calculated from data in article.

\section{COMMENTARY}

Bridging the transition from hospital to home in chronic heart failure appears to be essential for therapeutic success. A growing body of evidence supports the notion that such a bridge decreases morbidity from this disease, but cost effectiveness remains unclear. Home health care can be expensive and challenging and may lack standardisation, which results in heterogeneity of effectiveness from location to location. New approaches, such as the use of remote telemetry programmes that transmit patient information to a central facility, are now emerging to address these concerns.

In the study by Blue $e t a l$, an important finding that might affect the interpretation of the results relates to group differences in prescription of key drugs that occurred during the hospital stay. The intervention group had an absolute increment of $38 \%$ in the prescription of angiotensin converting enzyme inhibitors during the hospital stay, whereas the control group had only a $21 \%$ increase. In addition, the absolute dose in enalapril equivalents was 2-fold higher in the intervention group at hospital discharge. Even with this difference, the specialist nursing home care component was effective given that the magnitude and rapidity of benefit could not be explained solely by the pre-discharge drug intervention.

One final point relates to the population of patients admitted to hospital each year for heart failure secondary to diastolic dysfunction (an exclusion criterion in this study). To date, this distinct population has not been studied in clinical trials. No standard strategies for their care exist, even though this group might benefit more from lifestyle changes, medication, and strategies ensuring compliance.

Mandeep R Mehra, MD Ochsner Clinic Foundation New Orleans, Louisiana, USA

1 Mehra MR, Uber PA, Chomsky DB, et al. Emergence of electronic home monitoring in chronic heart failure: rationale, feasibility and early results with the Hommed "Sentry Observer" System. Congest Heart Fail 2000;6:137-9. 